\title{
Clinical and molecular characterization of (a) CrossMark POU3F4 mutations in multiple DFNX2 Chinese families
}

Yu Su, ${ }^{1,2}$, Xue Gao ${ }^{1,4+}$, Sha-Sha Huang ${ }^{1 \dagger}$, Jing-Ning Mao ${ }^{3}$, Bang-Qing Huang ${ }^{2}$, Jian-Dong Zhao ${ }^{1}$, Dong-Yang Kang ${ }^{1}$, Xin Zhang ${ }^{1}$ and Pu Dai ${ }^{1 *}$ (D)

\begin{abstract}
Background: Many X-linked non-syndromic hearing loss ( $\mathrm{HL}$ ) cases are caused by various mutations in the POU domain class 3 transcription factor 4 (POU3F4) gene. This study aimed to identify allelic variants of this gene in two Chinese families displaying X-linked inheritance deafness-2 (DFNX2) and one sporadic case with indefinite inheritance pattern.

Methods: Direct DNA sequencing of the POU3F4 gene was performed in these families and in 100 Chinese individuals with normal hearing.

Results: There are characteristic imaging findings in DFNX2 Chinese families with POU3F4 mutations. The temporal bone computed tomography (CT) images of patients with DFNX2 are characterized by a thickened stapes footplate, hypoplasia of the cochlear base, absence of the bony modiolus, and dilated internal acoustic meatus (IAM) as well as by abnormally wide communication between the IAM and the basal turn of the cochlea. We identified three causative mutations in POU3F4 for three probands and their extended families. In family 1468, we observed a novel deletion mutation, c.973delT, which is predicted to result in a p.Trp325Gly amino acid frameshift. In family 2741, the mutation c. 927delCTC was identified, which is predicted to result in the deletion of serine at position 310. In both families, the mutations were located in the POU homeodomain and are predicted to truncate the C-terminus of the POU domain. In the third family, a novel de novo transversion mutation (c.669 T > A) was identified in a 5-year-old boy that resulted in a nonsense mutation (p.Tyr223*). The mutation created a new stop codon and is predicted to result in a truncated POU3F4 protein.
\end{abstract}

Conclusions: Based on characteristic radiological findings and clinical features, POU3F4 gene mutation analysis will increase the success rate of stapes operations and cochlear implantations, and improve molecular diagnosis, genetic counseling, and knowledge of the molecular epidemiology of HL among patients with DFNX2.

Keywords: POU3F4, DFNX2, X-linked deafness, Mutation

\section{Background}

Hearing loss (HL) affects 1-3 per 1000 newborns, and the majority of congenital cases of HL are attributable to genetic factors [1]. Previous studies have indicated that deafness is transmitted with an inheritance pattern consistent with autosomal recessive transmission in $75-77 \%$

\footnotetext{
* Correspondence: daipu301@vip.sina.com

${ }^{\dagger}$ Yu Su, Xue Gao and Sha-Sha Huang contributed equally to this work.

${ }^{1}$ Department of Otorhinolaryngology, Head and Neck Surgery, PLA General

Hospital, Beijing 100853, People's Republic of China

Full list of author information is available at the end of the article
}

of cases and with autosomal dominant transmission in $15-20 \%$ of cases; $2-3 \%$ of human hereditary HL is caused by X-linked mutations [2, 3].To date, six deafness loci (DFNX1-6) have been mapped to chromosome X, with five of the corresponding genes identified: PRPS1 for DFNX1 [4], POU3F4 for DFNX2 [5], SMPX for DFNX4, AIFM1 for DFNX5 [6] and COL4A6 for DFNX6 [7-9] . PRPS1 on Xq22 encodes phosphoribosyl pyrophosphate synthetase 1; POU3F4 on Xq21 encodes a member of a transcription factor family that contains a POU domain; SMPX on Xp22 encodes the small muscle

(c) The Author(s). 2018 Open Access This article is distributed under the terms of the Creative Commons Attribution 4.0 International License (http://creativecommons.org/licenses/by/4.0/), which permits unrestricted use, distribution, and reproduction in any medium, provided you give appropriate credit to the original author(s) and the source, provide a link to the Creative Commons license, and indicate if changes were made. The Creative Commons Public Domain Dedication waiver (http://creativecommons.org/publicdomain/zero/1.0/) applies to the data made available in this article, unless otherwise stated. 
protein; and the recently identified gene COL4A6 encodes the alpha- 6 chain of collagen type IV at Xp21.

DFNX2, X-linked deafness type 2, is the most common type of X-linked $\mathrm{HL}$ in humans. Mutations in the POU3F4 gene were first found in 1995 [5] following its chromosomal localization to the X chromosome in 1988 [10]. In fact, deafness caused by POU3F4 mutation accounts for nearly $50 \%$ of all cases of X-linked non-syndromic HL $[5,11]$. Clinical features of DFNX2 often include mixed, progressive $\mathrm{HL}$, temporal bone anomalies, and stapes fixation [12, 13]. Affected males exhibit either mixed deafness or, less commonly, only sensorineural deafness. The temporal bone computed tomography (CT) image is characterized by a thickened stapes footplate, hypoplasia of the cochlear base, absence of the bony modiolus, and dilated internal acoustic meatus (IAM), as well as abnormally wide communication between the IAM and the basal turn of the cochlea [14]. Nance et al. first reported X-linked mixed deafness with congenital stapes fixation and a perilymphatic gusher [15]. Sennaroglu et al. suggested that the radiologic phenotype "X-linked deafness with stapes gusher" be called "incomplete partition type III" (IP- III) [16].

To date, nearly 60 different mutations in the coding region of the POU3F4 gene, including deletions, inversions, and duplications, have been reported to be associated with non-syndromic HL in families with DFNX2 (Table 1). Several reports have described mutations of POU3F4 in patients with HL and temporal bone abnormalities. In this report, we describe the clinical features and genetic analysis of two Chinese families displaying X-linked inheritance HL and one sporadic case with indefinite inheritance pattern. Moreover, two novel mutations (including a de novo mutation) were identified in the POU-specific and homeodomains of POU3F4, coinciding with familial HL.

\section{Methods}

\section{Clinical evaluation}

Patients were enrolled from families 1486, 2741, and ZSJ (three ethnic Han Chinese families) through the Department of Otolaryngology of the General Hospital of the People's Liberation Army, Beijing, China which collected data and DNA samples from more than 10,000 patients. We chose three families with specific manifestation of temporal bone CT from this cohort. Clinical evaluations, temporal bone imaging results, audiograms, and other relevant clinical information were collected for each family member. The probands had no obvious syndromic symptoms, and GJB2, SLC26A4, and m.1555A > $\mathrm{G}$ and $\mathrm{m} \cdot 1494 \mathrm{C}>\mathrm{T}$ mutations in mtDNA $12 \mathrm{~S}$ rRNA were excluded. Genomic DNA was extracted from peripheral blood using a blood DNA extraction kit according to the protocol provided by the manufacturer (Tiangen Biotech, Beijing, China).

\section{Families 1486, 2741, and ZSJ}

Family 1486 is a four-generation Chinese family, and the pedigree of this family is consistent with an X-linked inheritance pattern (Fig. 1b). Fifteen family members, including four patients (4 males: II:1, II:2, IV:3, and IV:4) with HL and 11 individuals (5 males: II:5, III:1, III:4, III:6, and IV:1, and 6 females: II:4, III:3, III:5, IV:2, IV:5, and IV:6) with normal hearing participated. The medical histories of the participants were obtained through structured questionnaires, otological examinations, and systematic assessments for signs of syndromic deafness. The proband underwent temporal bone CT scans and auditory brainstem responses. Pure tone audiometry was not available for the proband because of his young age.

Family 2741 is also a four-generation Chinese family, and the pedigree of this family showed a typical X-linked recessive inheritance pattern of HL (Fig. 2a). Ten family members were assessed: three patients (three males: II:3, III:9, and IV:2) with HL and seven individuals (two males: II:6 and III:13, and five females: II:5, II:7, II:12, III:10, and III:12) with normal hearing. The medical histories of the participants were obtained through structured questionnaires, otological examinations, and systematic assessments for signs of syndromic deafness. We obtained 10 blood samples (II:3, II:5, II:7, II:6, II:12, III:9, III:10, III:12, III:14, and IV:2) and the affected boy (IV:2) and his uncle (III:9) underwent temporal bone CT scans. Due to long distances, no temporal bone CT image was obtained from the affected boy's granduncle (II:3). The proband presented with mixed HL, his uncle had profound sensorineural HL, and the females presented with normal hearing.

In family ZSJ, the proband was a 5-year-old boy with congenital inner ear malformation and profound HL, but no one else in his family had HL, the inheritance pattern is unclear (Fig. 3e). Because of minimal progression in auditory ability after wearing hearing aid for 3 years and profound HL, the boy underwent cochlear implant surgery on his left ear at Chinese PLA General Hospital. A physical and otoscopic examination, temporal bone $\mathrm{CT}$ scans, and audiological studies were performed before surgery. According to the radiological and hearing findings, we suspected that he had DFNX2, and a mutation analysis of the POU3F4 gene was performed.

\section{Sequencing analysis of POU3F4}

Genomic DNA was extracted from blood using a DNA Extraction Kit (Tiangen Biotech). Briefly, the entire coding region and splice sites of the single exon of POU3F4 (NM_000307.1) were amplified in three overlapping 
Table 1 Reported Mutations in the POU3F4 Gene Resulting in DFNX2 Phenotypes

\begin{tabular}{|c|c|c|c|c|c|c|}
\hline Nucleotide change & Amino acid change $e^{a}$ & Protein domain ${ }^{b}$ & Feature of deafness ${ }^{c}$ & $\begin{array}{l}\text { Defects on } \\
\text { temporal bone } C T\end{array}$ & Location & References \\
\hline del 2.6 kb, 6.5 kb, 7 kb, 4.4 kb & NA & U & NA & NA & Korea & {$[22]$} \\
\hline del $8 \mathrm{~kb}$ & NA & U & Mixed & Yes & Korea & [22] \\
\hline de30 kb & NA & U & Mixed & Yes & Korea & {$[22]$} \\
\hline del $20 \mathrm{~kb}$ & NA & U & Mixed & Yes & Korea & [22] \\
\hline del $130 \mathrm{~kb}$ & NA & U & Mixed & Yes & Korea & {$[22]$} \\
\hline del $200 \mathrm{~kb}$ & NA & U & Mixed & Yes & Korea & {$[22]$} \\
\hline del $220 \mathrm{~kb}$ & NA & $U$ & Mixed & Yes & Korea & [22] \\
\hline del530 kb & NA & U & SNHL & Yes & US & {$[23]$} \\
\hline del $1200 \mathrm{~kb}$ & NA & $U$ & SNHL & Yes & Spain & [24] \\
\hline del entire gene & NA & Entire gene & Mixed & Yes & Korea & [22] \\
\hline c. $79 \mathrm{C}>\mathrm{T}$ & p.G $\ln 27^{*}$ & $U$ & SNHL & Yes & Poland, & [19] \\
\hline c. $293 C>A$ & p.Ser98* & $U$ & Mixed & Yes & France & [26] \\
\hline c.341G > A & p.Trp114* & U & $\mathrm{SNHL}$ & Yes & Pakistan & [27] \\
\hline c.346delG & p.Ala116Profs & U & $\mathrm{SNHL}$, Mixed & Yes & Poland,Turkey & {$[19,28]$} \\
\hline c.383delG & p.Gly 128 fs & U & SNHL & Yes & Korea & {$[29]$} \\
\hline c. $406 C>T$ & p.Gln136* & U & SNHL & Yes & Pakistan & {$[27]$} \\
\hline c.499 C > T & p.Arg167* & U & Mixed & Yes & Korea & [30] \\
\hline C. $.530 C>A$ & p.Ser $177^{*}$ & U & SNHL & Yes & China & [31] \\
\hline c.559G > T & p.Glu187* & U & $\mathrm{SNHL}$ & Yes & Poland, & {$[19]$} \\
\hline 601-606delTTCAAA & p.Phe201/Lys202 del & S & Mixed & Yes & Japan & {$[32]$} \\
\hline 603-610delCAAA & p.Lys202 fs & S & SNHL & Yes & Netherlands & {$[5]$} \\
\hline c.623 T > A & p. Leu208* & S & SNHL & Yes & Poland,Korea & {$[19,29,33]$} \\
\hline c. $632 C>T$ & p.Thr211Met & S & Mixed & Yes & Korea & [33] \\
\hline c.647G > A & p.Gly216 Glu & S & SNHL & Yes & China & {$[11]$} \\
\hline c.648-651delG & p.Arg215 fs & S & Mixed & Yes & Netherlands & {$[5]$} \\
\hline c.650 T > A & p.Leu217* & S & SNHL & Yes & Poland & [19] \\
\hline c.669 T > A & p.Tyr223* & S & SNHL & Yes & China & Present study \\
\hline c. $683 \mathrm{C}>\mathrm{T}$ & p.Ser228Leu & S & $\mathrm{SNHL}$ & Yes & US & {$[23]$} \\
\hline c. $686 \mathrm{~A}>\mathrm{G}$ & p.GIn229Arg & S & $\mathrm{SNHL}$ & Yes & Korea & [33] \\
\hline c. $689 \mathrm{C}>\mathrm{T}$ & p.Thr230lle & S & Mixed & Yes & US & [34] \\
\hline c.707A >C & p.Glu236Ala & S & $\mathrm{SNHL}$ & Yes & Turkey & {$[28]$} \\
\hline NA & p.Glu236Asp & S & NA & No & France & {$[26]$} \\
\hline c.727_728insA & p.Asn244Lysfs*26 & S & SNHL & Yes & Japan & [18] \\
\hline NA & p.Arg282Gln & $\mathrm{H}$ & NA & No & France & {$[26]$} \\
\hline NA & p.lle285Asn & $\mathrm{H}$ & NA & NA & France & {$[26]$} \\
\hline c.772delG & p. Glu 258Argfs & $\mathrm{H}$ & SNHL & Yes & Turkey & {$[28]$} \\
\hline c. $823 \mathrm{C}>\mathrm{T}$ & p.Gln $275^{*}$ & $\mathrm{H}$ & SNHL & Yes & Poland & [19] \\
\hline c.862del4 & p.Ser288Gln fs*37 & $\mathrm{H}$ & Mixed & Yes & UK & [35] \\
\hline NA & p.Ser288Cys fs*40 & $\mathrm{H}$ & NA & No & France & [26] \\
\hline c.895delA & p.Leu298 fs & $\mathrm{H}$ & Mixed & Yes & Netherlands & {$[5]$} \\
\hline$c .902 C>T$ & p.Pro301Leu & $\mathrm{H}$ & SNHL & NA & Ecuador & [28] \\
\hline$c .907 C>T$ & p.Pro303Ser & $\mathrm{H}$ & Mixed & Yes & UK & {$[25]$} \\
\hline c.916C > T & p.Gln306* & $\mathrm{H}$ & SNHL & Yes & Poland, & [19] \\
\hline
\end{tabular}


Table 1 Reported Mutations in the POU3F4 Gene Resulting in DFNX2 Phenotypes (Continued)

\begin{tabular}{|c|c|c|c|c|c|c|}
\hline Nucleotide change & Amino acid change ${ }^{a}$ & Protein domain ${ }^{b}$ & Feature of deafness ${ }^{c}$ & $\begin{array}{l}\text { Defects on } \\
\text { temporal bone CT }\end{array}$ & Location & References \\
\hline C.923 T > A & p.lle308Asn & $\mathrm{H}$ & Mixed & Yes & France & [26] \\
\hline NA & p.lle308 lle fs*28 & $\mathrm{H}$ & NA & No & France & [26] \\
\hline c.925 T>C & p.Ser309Pro & $\mathrm{H}$ & SNHL & Yes & China & [36] \\
\hline c.927delCTC & p.Ser310del & $\mathrm{H}$ & Mixed & Yes & Korea, China & $\begin{array}{l}\text { [37] } \\
\text { Present study }\end{array}$ \\
\hline c. $935 \mathrm{C}>\mathrm{T}$ & p.Ala312Val & $\mathrm{H}$ & SNHL & Yes & UK & [35] \\
\hline c.950 T> G & p.Leu317Trp & $\mathrm{H}$ & Mixed & Yes & Netherlands & [5] \\
\hline c.950dupT & p. Leu317Phefs*12 & $\mathrm{H}$ & SNHL & Yes & Korea & [33] \\
\hline c. $967 C>G$ & p.Arg323Gly & $\mathrm{H}$ & Mixed & Yes & Korea & [38] \\
\hline$c .971 \mathrm{~T}>\mathrm{A}$ & p.Val324Asp & $\mathrm{H}$ & SNHL & Yes & Poland, & [19] \\
\hline c.973delT & p.Trp325Glyfs*12 & $\mathrm{H}$ & Mixed & Yes & China & Present study \\
\hline c. $973 \mathrm{~T}>\mathrm{A}$ & p.Trp325Arg & $\mathrm{H}$ & SNHL & Yes & Germany & [39] \\
\hline c. $983 \mathrm{~A}>\mathrm{C}$ & p.Asn328Thr & $\mathrm{H}$ & Mixed & Yes & UK & [25] \\
\hline c. $985 C>$ G & p.Arg329Gly & $\mathrm{H}$ & Mixed & Yes & US & [34] \\
\hline c.986G > C & p.Arg329Pro & $\mathrm{H}$ & Mixed & Yes & Korea & [37] \\
\hline c.987 T > C & p.Leu308Thr & $\mathrm{H}$ & SNHL & NA & Nigeria & [28] \\
\hline c. $990 \mathrm{~A}>\mathrm{T}$ & p.Arg330Ser & $\mathrm{H}$ & SNHL & Yes & Netherlands & [5] \\
\hline c. $1000 A>G$ & p.Lys334Glu & $\mathrm{H}$ & Mixed & Yes & Netherlands & [5] \\
\hline c.1069delA & p. Thr 354GInfs*115 & $D$ & SNHL & Yes & Korea & [33] \\
\hline C. $1084 \mathrm{~T}>\mathrm{C}$ & p.X362Argexf*113 & $D$ & SNHL & Yes & Korea & [33] \\
\hline
\end{tabular}

${ }^{\mathrm{a}}{ }_{\mathrm{f}}$ frameshift, NA not available, ${ }^{\mathbf{b}} \mathrm{H}$ and $\mathrm{S}$ indicate the POU-homeodomain and POU-specific domain respectively; U Upstream, $D$ Downstream, ${ }^{\mathrm{C}} \mathrm{S} N H L$ sensorineural $\mathrm{HL}$, Mixed mixed HL

fragments using the following three pairs of forward $(\mathrm{F})$ and reverse (R) primers: 5'-ACTTCCTGCTTGGGTC TCATTG (F1) and 5'-GGAGTGATCCTGGCAATGGT (R1), 5'-GGCACCGAACCCGTCTATC (F2) and 5'-TC CCCTGGCGGAGTCAT (R2), and 5'-TTGGAGAAG GAAGTGGTGCG (F3) and 5'-CCCAGCTTGGACTG CTTAATGTA (R3). Polymerase chain reaction (PCR) amplification was performed in a total volume of $20 \mu \mathrm{L}$ : $2 \mu \mathrm{L}$ of $10 \times$ buffer, $0.5 \mu \mathrm{L}$ of primer $\mathrm{L}, 0.5 \mu \mathrm{L}$ of primer $\mathrm{R}, 0.5 \mu \mathrm{L}$ of deoxynucleotide triphosphates, $0.2 \mu \mathrm{L}$ of Taq polymerase, $1 \mu \mathrm{L}$ of DNA, and $15.3 \mu \mathrm{L}$ of water. PCR began with incubation at $95{ }^{\circ} \mathrm{C}$ for $5 \mathrm{~min}$, followed by nine cycles of denaturation for $45 \mathrm{~s}$ at $95{ }^{\circ} \mathrm{C}$, annealing for $45 \mathrm{~s}$ at $58{ }^{\circ} \mathrm{C}$, and extension for $30 \mathrm{~s}$ at $72{ }^{\circ} \mathrm{C}$; this was followed by 34 cycles of denaturation for $45 \mathrm{~s}$ at $95{ }^{\circ} \mathrm{C}$, annealing for $45 \mathrm{~s}$ at $55{ }^{\circ} \mathrm{C}$, extension for $30 \mathrm{~s}$ at $72{ }^{\circ} \mathrm{C}$, and a final 7 -min extension at $72{ }^{\circ} \mathrm{C}$. PCR products were resolved by gel electrophoresis to confirm product amplification. Sequencing was performed using the ABI 3100 Avant Capillary Electrophoresis System (Applied Biosystems, Foster City, CA, USA). We also sequenced 100 Chinese individuals with normal hearing to determine whether the mutations were present in the unaffected Chinese population.

\section{Results}

\section{Clinical features of the family}

In family 1486 , the proband and the affected male members exhibited congenital severe-to-profound sensorineural hearing impairment. Auditory brainstem response thresholds with clicks were $70 \mathrm{~dB}$ in both ears of the proband. In family 2741, the proband exhibited typical audiometric features of mixed hearing impairment (Fig. 2b), while the maternal uncle of the proband was completely deaf (Fig. 2d). In family ZSJ, the proband exhibited profound sensorineural deafness (Fig. 3a).

Temporal bone computed tomography (CT) was performed in four patients from three families; identical findings were observed: an absent modiolus, and the basal turn of the cochlea was incompletely separated from the IAM and appeared to be a continuation of the IAM. Typically, a bulbous dilatation of the lateral end of the IAM was identified (Figs. 1a, 2c, e, and 3b: R) (arrow). All of the findings were symmetrical, except that the left cochlea of the affected boy in family ZSJ was not fully developed and smaller than the cochlea of the other side (Fig. 3e).

Cochlear implantation with the Nucleus ${ }^{\circ}$ Slim Straight Electrode was performed in the proband from family 


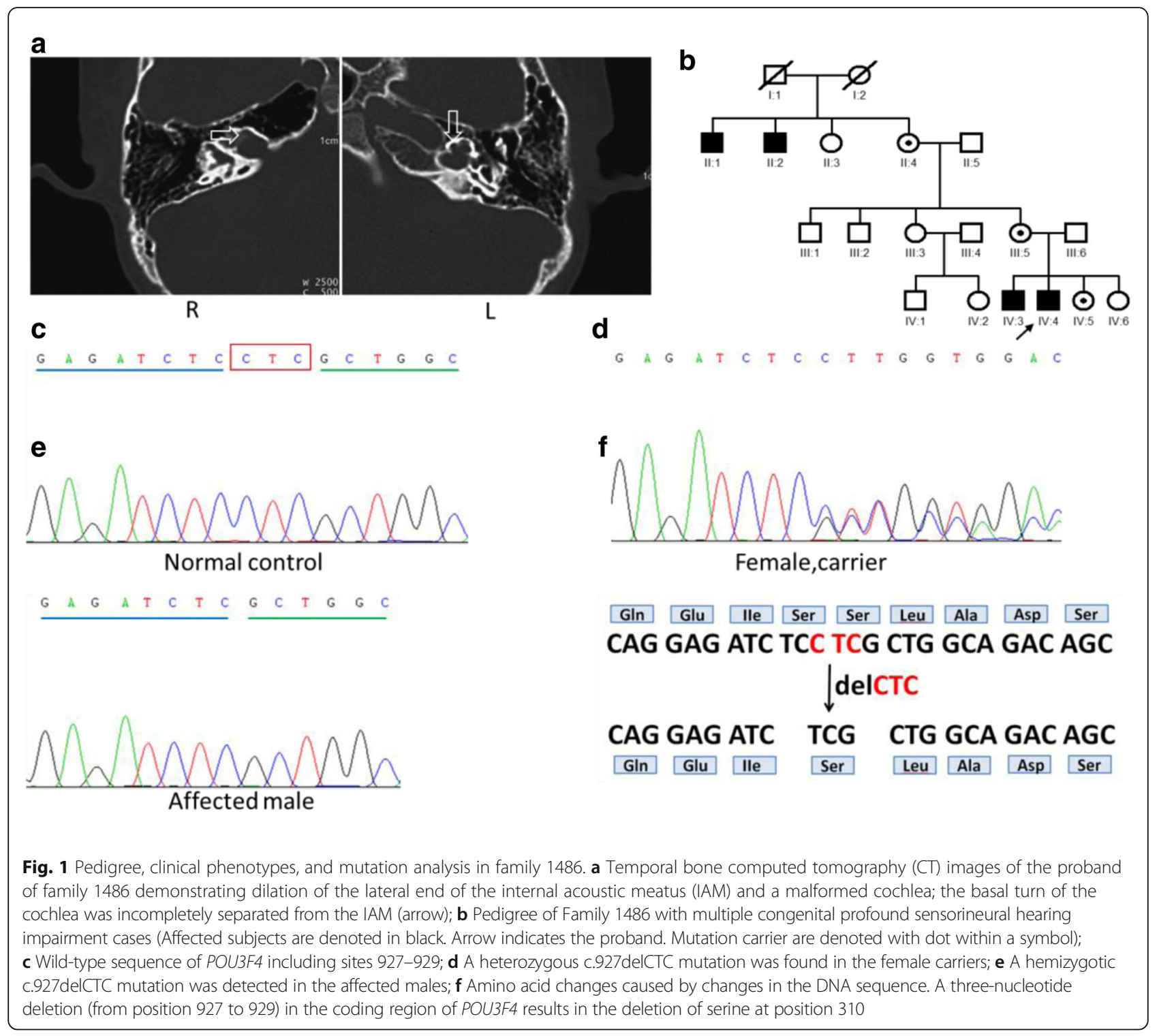

ZSJ. Due to widening of the bony IAM during cochlear implantation, cerebrospinal fluid (CSF) "gusher" was observed upon opening the round window, and a piece of prepared muscle tissue was used to block the leakage after inserting the electrodes. CSF leakage did not occur after surgery in this case. Intra-operative $\mathrm{CT}$ was utilized to ensure correct electrode positioning and to prevent the electrode from entering the IAM. The postoperative pure tone audiograms under aided conditions showed hearing thresholds of $60 \mathrm{~dB}$.

\section{Identification of POU3F4 mutations in families 1486,} 2741, and ZSJ with DFNX2

In family 1486, the c.927delCTC (p.Ser310del) mutation was identified in the males (IV:3 and IV:4) with profound $\mathrm{HL}$, consistent with X-linked inheritance. A three-nucleotide deletion (from position 927 to 929) in the coding region of POU $3 F 4$ resulted in the deletion of a serine at position 310 within the POU homeodomain (Fig. 1e), without affecting the coding frame (Fig. 1f). The mother (III:5), grandmother (II:4), and sister (IV:5) were heterozygous for c.927delCTC (Fig. 1d).

In family 2741, the 10 family members were enrolled in the study, 3 of whom were classified as affected (consistent with X-linked inheritance) (Fig. 2a). The c.973delT (p.Trp325Glyfs*12) mutation was identified in these three male patients, leading to a predicted frameshift mutation and truncation of the protein. This mutant protein lacks part of the POU3F4 protein, including the POU homeodomain, which is highly conserved across species (Fig. 2j). Sequence analysis of all family members revealed a deletion at nucleotide position 973 


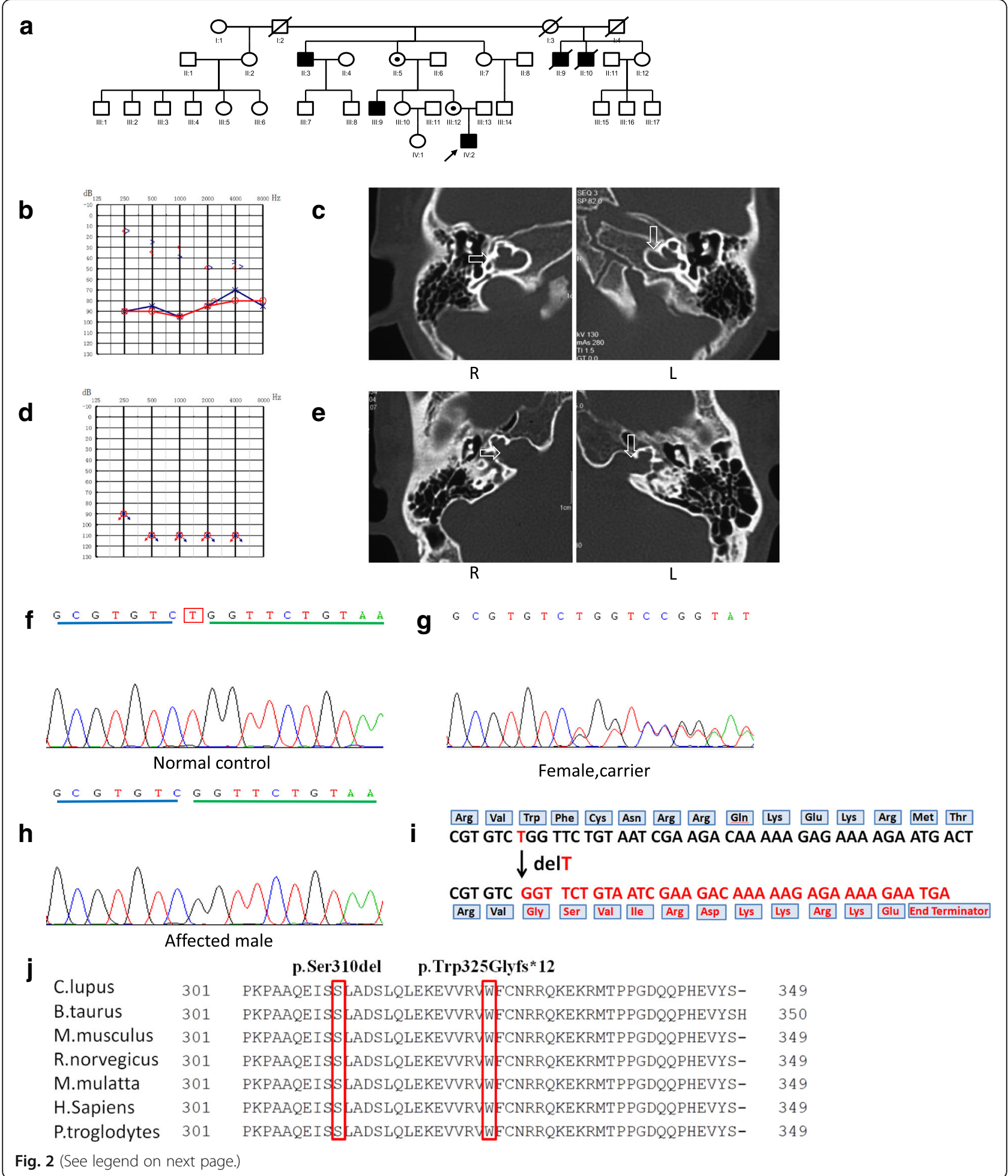


(See figure on previous page.)

Fig. 2 Pedigree, clinical phenotypes and mutation analysis in family 2741. a Pedigree of family 2741 with congenital mixed hearing impairment and sensorineural hearing impairment cases (Affected subjects are denoted in black. Arrow indicates the proband. Mutation carrier are denoted with dot within a symbol); b Audiograms of both ears for the proband, who exhibited typical audiometric features of mixed hearing impairment; c Temporal bone CT images of the proband demonstrating dilation of the bottom of the IAM and a deficit in the bony plate, which separates the basal turn of the cochlea and the IAM (arrow); d Audiograms of both ears from the uncle of the proband, who shows profound sensorineural hearing impairment; e Temporal bone CT images of the uncle of the proband demonstrating dilation of the lateral end of the IAM and bone deficiency between the basal turn of the cochlea and the IAM (arrow). $\mathbf{f}$ Wild-type sequence of POU3F4 including position 973; $\mathbf{g}$ A heterozygous c.973delT mutation was found in the female carriers; $\mathbf{h}$ A hemizygotic c.973delT mutation was detected in the affected males; $\mathbf{i}$ Amino acid change caused by changes in the DNA sequence leading to a predicted frameshift mutation and truncation of the POU3F4 protein; $\mathbf{j}$ Panel 1 marks the position of the c.973delT (p.Trp325Glyfs*12) mutation and panel 2 marks the position of the c.927delCTC (p.Ser310del) mutation. The POU homeodomain (from Gly276 to Arg335) is highly conserved in different species

in the patient (IV:2, II:3 and III:9; Fig. 2h), and the mother (III:12) and grandmother (II:5) were heterozygous for c.973delT (Fig. 2g). Mutation screening of POU3F4 in families 2741 and 1486 showed that p.Trp325Glyfs"12 and p.Ser310del, respectively, co-segregated in all affected males examined. Heterozygous p.Trp325Glyfs*12 and p.Ser310del were also found in the female carriers, separately. Moreover, neither mutation was observed in any of the 100 unrelated controls with normal hearing by direct sequencing. According to the standards and guidance in

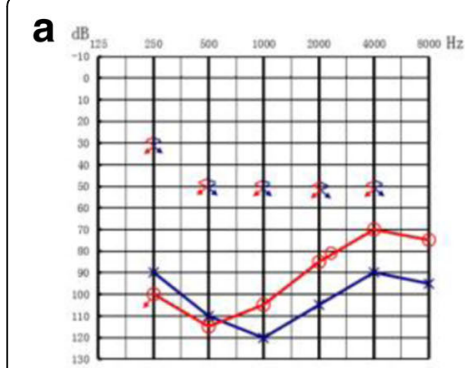

C
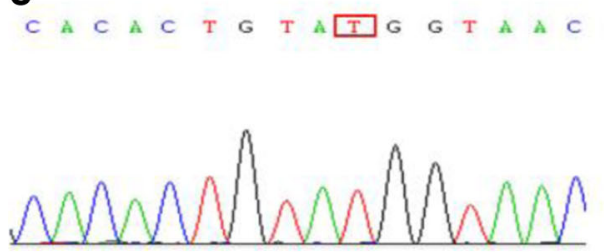

Normal control (Father and Mother)

f

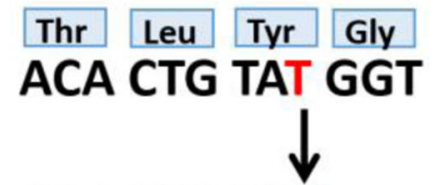

ACA CTG TAA

Thr Leu End Terminator

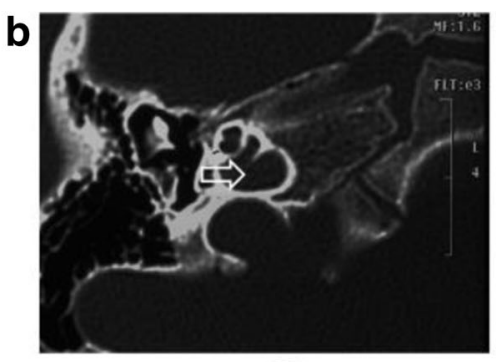

$\mathrm{R}$

d

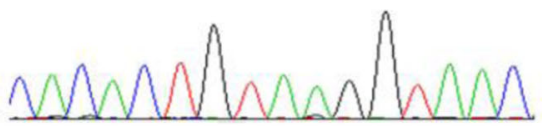

Affected boy

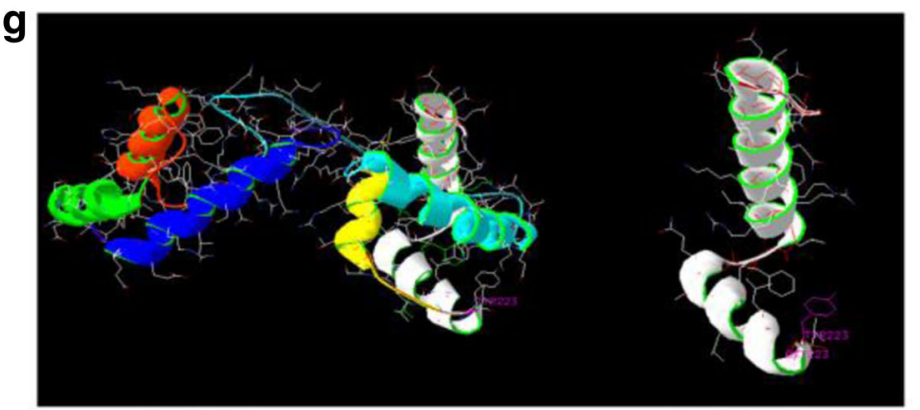

Wild-type

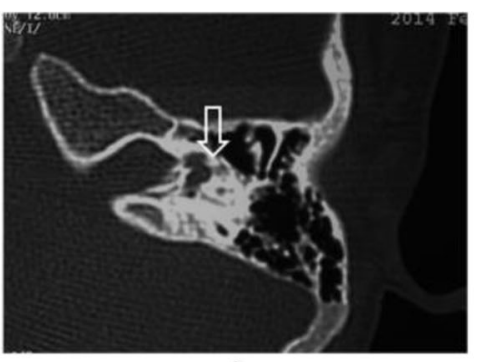

$\mathrm{L}$

e

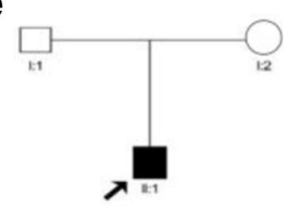

p.Try223*

Fig. 3 Pedigree, clinical phenotypes and mutation analysis in family ZSJ. a Audiograms of both ears from the proband exhibited profound sensorineural hearing impairment; $\mathbf{b}$ Temporal bone CT images of the proband demonstrating dilation of the lateral end of the IAM and a deficit in the basal turn of the cochlea in the right ear (arrow) in addition to dilation of the lateral end of the IAM and an incompletely developed cochlea in the left ear (arrow); c Wild-type sequence of POU3F4, including site 669; d A hemizygotic c.669 T> A mutation was detected in the affected boy; e Pedigree of family ZSJ; $\mathbf{f}$ Stop codon caused by changes in the DNA sequence; $\mathbf{g}$ Molecular modeling of wild-type and mutant POU3F4 proteins. The c.669 T > A mutant creates a new stop codon and is predicted to result in a truncated protein lacking normal POU3F4 transcription factor function 


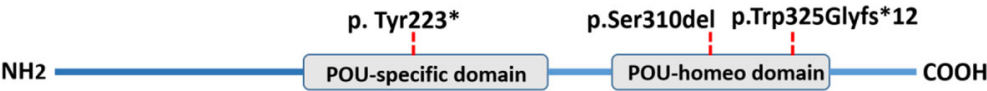

Fig. 4 Schematic illustration of the POU3F4 protein. In this study, three mutations were identified: the p.Tyr223*mutation located in the POUspecific domain, and the p.Trp325Glyfs*12 and p.Ser310del mutations located in the POU homeodomain

the 2015 American College of Medical Genetics and Genomics (ACMG), two variants identified in this study, c.973delT and c.927delCTC (p.Trp325Glyfs*12 and p.Ser310del), are pathogenic variants, not rare polymorphisms, and are among the most conserved amino acids in the POU homeodomain (Fig. 2j).

In family ZSJ, sequence analysis of POU $3 F 4$ in the affected boy revealed a de novo transversion, c.669 T $>$ A (Fig. 3d), resulting in a nonsense mutation (p.Tyr223*, Fig. 3f) and the creation of a new stop codon. Thus, it is predicted to result in a truncated protein lacking normal POU3F4 transcription factor function. Neither parent carried the c.669 T > A mutation (Fig. 3c). To determine the reliability of the de novo mutation, a paternity test was used to confirm the biological relationship between the parents and the boy. Moreover, the mutation was not found in any of the 100 unrelated controls with normal hearing by direct sequencing. Molecular modeling showed that the tyrosine residue at position 223 is located at the end of the second helix of the specific homeodomain. The c.669 T > A mutant is predicted to result in the deletion of the third helix of the specific homeodomain and all three helices of the POU homeodomain, which creates a truncated protein lacking normal POU3F4 transcription factor function (Fig. 3g)

Two single nucleotide polymorphisms, Ala708Gly and Gly710Cys (rs5921978 and rs5921979, respectively), were also observed in all subjects of the families and controls, indicating polymorphisms (data not shown).

In this study, three mutations were identified: the p.Tyr223* mutation located in the POU specific-domain, and the p.Trp325Glyfs*12 and p.Ser310del mutations located in the POU homeodomain (Fig. 4).

\section{Discussion}

Deafness segregating at the DFNX2 locus is associated with mutations in the POU3F4 gene. The human POU3F4 protein contains a POU-specific domain, with a length of 67 amino acids (from Lys194 to Asp260), a linker of 15 residues (from Ser261 to Gln275), and a POU homeodomain, with a length of 60 amino acids (from Gly276 to Arg335) [17]. Previous studies have suggested that HL in DFNX2 is caused by loss-of-function of the POU3F4 protein rather than gain-of-ectopic functions in the mutant proteins.

Previous studies have identified more than 60 mutations invariably located in the POU- specific and homeodomains of POU3F4. In this study, we identified three mutations in the POU3F4 gene in two Chinese families displaying $\mathrm{X}$-linked inheritance $\mathrm{HL}$ and one sporadic case with indefinite inheritance pattern. Two of the mutations (p.Trp325Glyfs*12 and p.Ser310del) occurred in the POU homeodomain and the third mutation (p.Tyr223\%) was identified as a de novo mutation occurring in the specific homeodomain, which caused a premature termination resulting in a protein lacking part of the specific homeodomain and the entire POU homeodomain. Usually, diagnosing the disorder in sporadic cases with inner ear malformation is difficult. In this report, POU3F4 gene sequencing identified a "novel de novo" nonsense mutation (c.669 T > A). This is the third reported case in which such a disorder occurred in the affected individual due to a spontaneous de novo mutation not inherited from the parents. Two previously reported POU3F4 de novo mutations were p.Asn244Lysfs*26 [18] and p.Leu217* [19]. We believe that many more such patients with this disorder are likely to be diagnosed in the near future due to a combination of clinical features and genetic testing.

Individuals, usually males, with variants in this gene exhibit characteristic clinical and radiological features. The most frequent form of X-linked deafness, DFNX2, is characterized by temporal bone abnormalities, stapes fixation and, in most cases, a mixed type of deafness. All of the probands from the three families examined herein showed characteristic inner ear radiological features compatible with incomplete partition type III. The HL in these individuals can be mixed, with the sensorineural component usually presenting in infancy and showing progression with age. Temporal bone CT scans showed dilatation of the lateral end of the IAM and/or a bone deficiency between the basal turn of the cochlea and the IAM. The conductive HL component, which may or may not be present, is due to fixation of the stapes. Because of outward pressure of perilymphatic fluid on the oval window coupled with defects in the size and shape of the stapes footplate further compromise ossicular movement and, collectively, these anomalies result in progressive sensorineural deafness in patients with DFNX2. In patients with radiological abnormalities of the cochlea on CT scans like this, a perilymphatic flow (or "gusher") can occur during inner ear surgery [20], which will result in immediate deafness along with concomitant complaints of vertigo and tinnitus. Preoperative evaluation before stapes or cochlear implant surgery 
is very important. In our cases, an expected CSF gusher was seen in the patient (family ZSJ) when the round window was opened. Cochleostomy was sealed with muscle tissue, and there was no CSF leakage, meningitis, or facial stimulation after surgery. Saeed et al. encouraged additional surgical obliteration of the middle ear space and external auditory canal to avoid persistent CSF leakage and its associated complications [21]. However, in our cases, this was not necessary.

The management of patients with DFNX2 depends on the degree of the overall HL. If the HL is a milder conductive or sensorineural hearing impairment, hearing aids are often a first-line recommendation. Some patients with bilateral mixed HL but serviceable bone conduction thresholds benefit from bone-anchored hearing aid technologies. However, patients with severe-to-profound hearing impairment can benefit from cochlear implant surgery. Kang et al. compared the audiologic performance of patients with X-linked deafness after cochlear implantation to those with a normal inner ear structure after implantation and found no significant difference between the two groups. The patient who underwent cochlear implantation described in this article had postoperative hearing thresholds of approximately $60 \mathrm{~dB}$ at 12 and 24 months after activation of the cochlear implant. We believe that the limited auditory perception and language acquisition were due to serious malformation of the cochlea. Regardless, with thoughtful preparation and the assistance of intraoperative imaging, cochlear implantation in patients with DFNX2 can be performed safely.

Several studies have reported HL in female siblings or mothers of affected males with mutations in POU3F4. In 2009, Marlin et al. reported the phenotype of eight independent females from families which male carriers presenting with typical DFNX2 and carrying POU3F4 variants, and in which three female carriers have hearing loss [35]. However, we did not observe HL in the heterozygous mothers in our two families (families 1486 and 2741).

\section{Conclusions}

The identification of pathogenic alleles causing X-linked recessive deafness will improve molecular diagnosis, genetic counseling, and knowledge of the molecular epidemiology of HL among Chinese individuals. Taking these results together, we recommend preoperative gene mutation analysis in patients who have DFNX2 diagnosed on the basis of characteristic radiological findings. If a genetic cause of HL is determined, families with hereditary HL can be provided with prognostic information, the risk of recurrence, and improved rehabilitation options.

\section{Abbreviations}

ACMG: American College of Medical Genetics and Genomics; CT: Computed tomography; DFNX2: X-linked inheritance deafness-2; HL: Hearing loss; IAM: Internal acoustic meatus; POU3F4: POU domain class 3 transcription factor 4

\section{Acknowledgements}

We sincerely thank all the family members for their participation and cooperation in this study.

\section{Funding}

These investigations were supported by funding from the National Natural Science Foundation of China (81730029 and 81371096) and the National Key Research and Development Project (2016YFC1000700 and 2016YFC1000704) to PD, the China Postdoctoral Granted Financial Support and Special Financial Grant (Nos. 20120481482 and 201104779) and the Chinese National Nature Science Foundation Research Grant (No. 81400471) to YS, the Chinese National Nature Science Foundation Research Grant (No. 81570929) to XG, and the Chinese National Natural Science Foundation of China (81200751) to $\mathrm{SSH}$. Funders had no role in the study design, data collection and analysis, decision to publish, or preparation of the manuscript.

\section{Availability of data and materials}

Sequencing data of the affected subjects and their parents have been provided in the paper.

\section{Authors' contributions}

Conceived and designed the experiments: PD. Performed the experiments: YS, SSH, BQH, JDZ, and FY. Analyzed the data: YS, XG, and SSH. Contributed reagents/materials/analysis tools: $X Z$ and DYK. Wrote the paper: YS and PD. All authors have read and approved the final manuscript.

Ethics approval and consent to participate

This study was approved by the Chinese PLA General Hospital Research Ethics Committee.

\section{Consent for publication}

Fully informed written consent for participation and publication of the clinical data was obtained from each subject or from the guardians of subjects $<18$ years old.

\section{Competing interests}

The authors declare that they have no competing interests.

\section{Publisher's Note}

Springer Nature remains neutral with regard to jurisdictional claims in published maps and institutional affiliations.

\section{Author details \\ ${ }^{1}$ Department of Otorhinolaryngology, Head and Neck Surgery, PLA General Hospital, Beijing 100853, People's Republic of China. ${ }^{2}$ Department of Otorhinolaryngology, Hainan Branch of PLA General Hospital, Sanya 572000, People's Republic of China. ${ }^{3}$ Department of Medical Imaging, PLA 307 Hospital, Beijing 100074, People's Republic of China. ${ }^{4}$ Department of Otolaryngology, The General Hospital of the PLA Rocket Force, 16\# Xi Wai Da Jie, Beijing 100088, People's Republic of China.}

Received: 6 February 2017 Accepted: 21 June 2018

Published online: 04 September 2018

References

1. Morton CC, Nance WE. Newborn hearing screening-a silent revolution. N Engl J Med. 2006;354(20):2151-64.

2. Marazita ML, et al. Genetic epidemiological studies of early-onset deafness in the U.S. school-age population. Am J Med Genet. 1993;46(5):486-91.

3. Morton NE. Genetic epidemiology of hearing impairment. Ann N Y Acad Sci. 1991;630:16-31.

4. Liu X, et al. Loss-of-function mutations in the PRPS1 gene cause a type of nonsyndromic X-linked sensorineural deafness, DFN2. Am J Hum Genet. 2010;86(1):65-71.

5. de Kok YJ, et al. Association between X-linked mixed deafness and mutations in the POU domain gene POU3F4. Science. 1995;267(5198):685-8. 
6. Zong L, et al. Mutations in apoptosis-inducing factor cause X-linked recessive auditory neuropathy spectrum disorder. J Med Genet. 2015;52(8): 523-31

7. Huebner AK, et al. Nonsense mutations in SMPX, encoding a protein responsive to physical force, result in X-chromosomal hearing loss. Am J Hum Genet. 2011;88(5):621-7.

8. Schraders $M$, et al. Next-generation sequencing identifies mutations of SMPX, which encodes the small muscle protein, $X$-linked, as a cause of progressive hearing impairment. Am J Hum Genet. 2011;88(5):628-34.

9. Rost $\mathrm{S}$, et al. Novel form of X-linked nonsyndromic hearing loss with cochlear malformation caused by a mutation in the type IV collagen gene COL4A6. Eur J Hum Genet. 2014;22(2):208-15.

10. Wallis $C_{\text {, et }}$ al. X-linked mixed deafness with stapes fixation in a Mauritian kindred: linkage to Xq probe pDP34. Genomics. 1988;3(4):299-301.

11. Li J, et al. Identification of a novel mutation in POU3F4 for prenatal diagnosis in a Chinese family with X-linked nonsyndromic hearing loss. J Genet Genomics. 2010;37(12):787-93.

12. Cremers CW, Huygen PL. Clinical features of female heterozygotes in the $X$ linked mixed deafness syndrome (with perilymphatic gusher during stapes surgery). Int J Pediatr Otorhinolaryngol. 1983;6(2):179-85.

13. Phelps PD, et al. X-linked deafness, stapes gushers and a distinctive defect of the inner ear. Neuroradiology. 1991;33(4):326-30.

14. Gong WX, Gong RZ, Zhao B. HRCT and MRI findings in X-linked nonsyndromic deafness patients with a POU3F4 mutation. Int J Pediatr Otorhinolaryngol. 2014;78(10):1756-62.

15. Nance WE, et al. X-linked mixed deafness with congenital fixation of the stapedial footplate and perilymphatic gusher. Birth Defects Orig Artic Ser. 1971;07(4):64-9.

16. Sennaroglu L, Sarac S, Ergin T. Surgical results of cochlear implantation in malformed cochlea. Otol Neurotol. 2006;27(5):615-23.

17. Mathis JM, et al. Brain 4: a novel mammalian POU domain transcription factor exhibiting restricted brain-specific expression. EMBO J. 1992;11(7): 2551-61.

18. Moteki $\mathrm{H}$, et al. De novo mutation in X-linked hearing loss-associated POU3F4 in a sporadic case of congenital hearing loss. Ann Otol Rhinol Laryngol. 2015;124(Suppl 1):169S-76S.

19. Pollak A, et al. Novel and De Novo Mutations Extend Association of POU3F4 with Distinct Clinical and Radiological Phenotype of Hearing Loss. PLoS One. 2016;11(12):e0166618

20. Cremers CW, Hombergen GC, Wentges RT. Perilymphatic gusher and stapes surgery. A predictable complication? Clin Otolaryngol Allied Sci. 1983;8(4): 235-40.

21. Saeed H, Powell HR, Saeed SR. Cochlear implantation in X-linked deafness How to manage the surgical challenges. Cochlear Implants Int. 2016;17(4): $178-83$

22. de Kok YJ, et al. Identification of a hot spot for microdeletions in patients with X-linked deafness type 3 (DFN3) $900 \mathrm{~kb}$ proximal to the DFN3 gene POU3F4. Hum Mol Genet. 1996;5(9):1229-35.

23. Vore AP, et al. Deletion of and novel missense mutation in POU3F4 in 2 families segregating $X$-linked nonsyndromic deafness. Arch Otolaryngol Head Neck Surg. 2005;131(12):1057-63.

24. Arellano B, et al. Sensorineural hearing loss and Mondini dysplasia caused by a deletion at locus DFN3. Arch Otolaryngol Head Neck Surg. 2000;126(9): 1065-9

25. Cremers FP, Cremers CW, Ropers HH. The ins and outs of X-linked deafness type 3. Adv Otorhinolaryngol. 2000;56:184-95.

26. Marlin S, et al. Phenotype and genotype in females with POU3F4 mutations. Clin Genet. 2009;76(6):558-63.

27. Waryah AM, et al. Molecular and clinical studies of X-linked deafness among Pakistani families. J Hum Genet. 2011;56(7):534-40.

28. Bademci $G$, et al. Novel domain-specific POU3F4 mutations are associated with X-linked deafness: examples from different populations. BMC Med Genet. 2015;16:9.

29. Lee HK, et al. Novel POU3F4 mutations and clinical features of DFN3 patients with cochlear implants. Clin Genet. 2009;75(6):572-5.

30. Stankovic KM, et al. Cochlear implantation in children with congenital Xlinked deafness due to novel mutations in POU3F4 gene. Ann Otol Rhinol Laryngol. 2010;119(12):815-22.

31. Huang B-q, Zeng J-I, Su Y, Dai P. A novel POU3F4 gene mutation for Xlinked recessive hereditary hearing loss. Chinese J Otology. 2014;12(1):57-60.
32. Hagiwara $\mathrm{H}$, et al. A new mutation in the POU3F4 gene in a Japanese family with X-linked mixed deafness (DFN3). Laryngoscope. 1998;108(10): 1544-7.

33. Choi BY, et al. Destabilization and mislocalization of POU3F4 by C-terminal frameshift truncation and extension mutation. Hum Mutat. 2013;34(2):309-16.

34. Friedman RA, et al. Molecular analysis of the POU3F4 gene in patients with clinical and radiographic evidence of X-linked mixed deafness with perilymphatic gusher. Ann Otol Rhinol Laryngol. 1997;106(4):320-5.

35. Bitner-Glindzicz $M$, et al. Further mutations in Brain 4 (POU3F4) clarify the phenotype in the X-linked deafness, DFN3. Hum Mol Genet. 1995;4(8):1467-9.

36. Wang QJ, et al. A novel mutation of POU3F4 causes congenital profound sensorineural hearing loss in a large Chinese family. Laryngoscope. 2006; 116(6):944-50.

37. Lee HK, et al. Clinical and molecular characterizations of novel POU3F4 mutations reveal that DFN3 is due to null function of POU3F4 protein. Physiol Genomics. 2009;39(3):195-201.

38. de Kok YJ, et al. The molecular basis of X-linked deafness type 3 (DFN3) in two sporadic cases: identification of a somatic mosaicism for a POU3F4 missense mutation. Hum Mutat. 1997;10(3):207-11.

39. Schild C, et al. Novel mutation in the homeobox domain of transcription factor POU3F4 associated with profound sensorineural hearing loss. Otol Neurotol. 2011:32(4):690-4

\section{Ready to submit your research? Choose BMC and benefit from:}

- fast, convenient online submission

- thorough peer review by experienced researchers in your field

- rapid publication on acceptance

- support for research data, including large and complex data types

- gold Open Access which fosters wider collaboration and increased citations

- maximum visibility for your research: over $100 \mathrm{M}$ website views per year

At BMC, research is always in progress.

Learn more biomedcentral.com/submissions 